\title{
Inclination Dependent Luminosity Function of Spiral Galaxies
}

\author{
Zhengyi Shao \\ Shanghai Astronomical Observatory, Joint institute for galaxy \& cosmology, CAS, Shanghai \\ 200030, P.R. China \\ email: zyshao@shao.ac.cn
}

We study the inclination dependent luminosity function (LF) of spiral galaxies of the Sloan Digital Sky Survey (SDSS). Up to 60000 sample galaxies are selected from the 2nd data release of SDSS by fracde $_{r}<0.5$. Magnitudes and other related photometric parameters are taken from NYU-VAGC(Blanton et al. 2005). The apparent axis ratio $(b / a)$ is used as an observational inclination indicator to define sub-samples. LFs of all 5 SDSS bands $(u, g, r, i, z)$ are drawn for different sub-samples. Significant correlation is found between characteristic magnitudes $\left(M^{*}\right)$ of sub-samples and their inclinations, which can be fairly explained by dust extinction. A linear fit of the relation between $M^{*}$ and $\log (b / a)$ measures the $M^{*}(0)$ (for expected face-on spirals, with $0.2 \sim 0.3$ mag brighter than that of LF of whole sample) and the intensity of dust extinction $\gamma$, for each band (Figure 1(a)). Additionally, since $\gamma \propto \tau$ (optical depth), the wavelength dependent $\gamma$ describes the extinction curve. Figure 1(b) shows a good linear fit that implies the extinction curve obeys the power law very well, with $\tau_{\lambda}=\tau_{V}(\lambda / 5500 \AA)^{-0.97 \pm 0.07}$. The power index $n \sim 1$ is shallower than that of the MW, LMC and SMC $(n=1.1 \sim 1.5)$, but significantly steeper than the value of Charlot et al. (2000) $(n=0.7)$, which under the assumption of a patchy distribution of dust in spiral galaxies. So our result implies that dust distributed in spirals, on average, are not as patchy as Charlot et al. assumed.
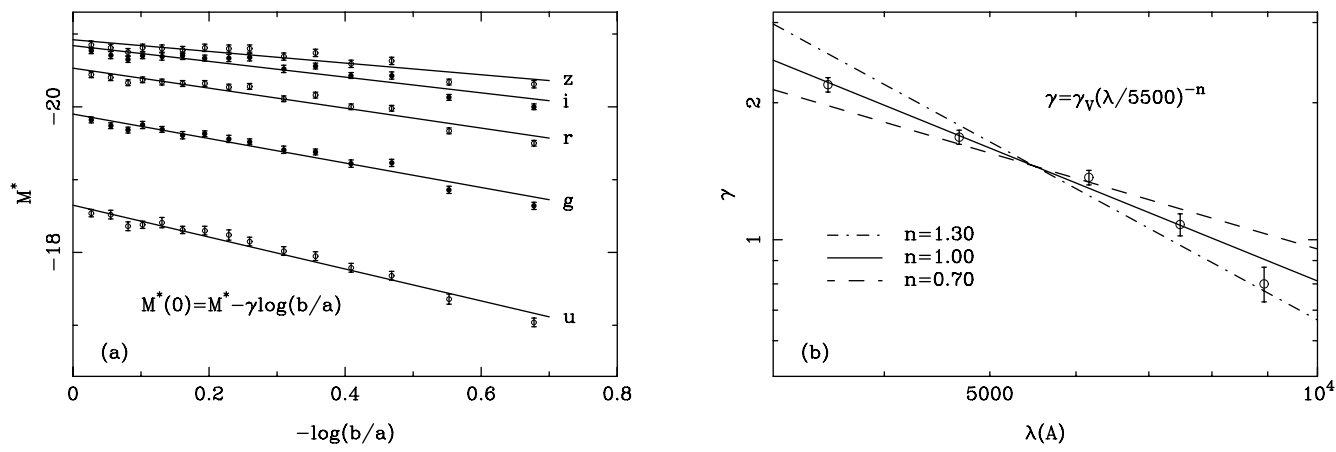

Figure 1. (a)Inclination dependent $M^{*}$ of SDSS spiral galaxies; (b)Extinction curve.

\section{Acknowledgements}

This research was supported by NSFC grants No.10273016, 10333060.

\section{References}

Blanton, M.R. et al., 2005, AJ, 129, 2562.

Charlot, S., \& Fall, S.M. 2000, ApJ, 539,718. 